\title{
A REVIEW OF CURRENT RESEARCH ON POWDER MIXED ELECTRIC
}

\section{DISCHARGE MACHINING}

\section{S. DEEPTHI ${ }^{1} \&$ B. V. DHARMENDRA ${ }^{2}$}

${ }^{I}$ Research Scholar, Department of Mechanical Engineering, Koneru Lakshmaiah Education Foundation,

Green Fields, Vadddeswaram, Guntur, India

${ }^{2}$ Professor, Department of Mechanical Engineering, Koneru Lakshmaiah Education Foundation,

Green Fields, Vadddeswaram, Guntur, India

\begin{abstract}
In Die Sink electric discharge machining, mostly the dielectric material is in the liquid state such as mineral oils, kerosene, transformer oil, electric discharge machining oils or synthetic oils. Inferior surface quality, higher tool wear ratio, lesser material removal rate and higher machining time are the main disadvantages of using these dielectric fluids in electric discharge machining. The use of powder mixed with dielectric fluid named as a powder mixed electric discharge machining (PMEDM) is dependable to overcome the above-mentioned disadvantages and electric discharge machining performance improvement,. The current paper presents a literature review on the usage and effects of powder mixed electric discharge machining in characteristics of electrical discharge machining.

KEYWORDS: Die Sink Electric Discharge Machining, Dielectric Fluid, Powder Mixed Electric Discharge Machining, Material Removal Rate
\end{abstract}

Received: Mar 12, 2019; Accepted: Apr 02, 2019; Published: Apr 24, 2019; Paper Id.: IJMPERDJUN201948

\section{ABBREVIATIONS}

$\begin{array}{ll}\text { EDM } & \text { Electric Discharge Machining } \\ \text { PMEDM } & \text { Powder Mixed Electric Discharge Machining } \\ \text { TOPSIS } & \text { The Technique for Order of Preference by Similarity to Ideal Solution } \\ \text { GRA } & \text { Grey Relational Analysis } \\ \text { ANOVA } & \text { Analysis of variance } \\ \text { MRR } & \text { Material Removal Rate } \\ \text { SR } & \text { Surface Roughness } \\ \text { TWR } & \text { Tool Wear Rate } \\ \text { RLT } & \text { Recast Layer Thickness } \\ \text { MMC } & \text { Metal Matrix Composite }\end{array}$

\section{INTRODUCTION}

The sinker electric discharge machining process utilizes a specifically designed geometry of an electrically charged electrode for burning the geometry into a metal part. In dies and molds production this machining process is commonly employed. A three-dimensional electrode is used to form the required geometry negatively in the metal. The variations in shapes, cavities, and indentions are created by superimposing movements in $\mathrm{x}, \mathrm{y}, \mathrm{c}, \mathrm{z}$, the main axes, like those cannot be achieved in part by any other machining system.[1] 


\subsection{Powder Mixed Electric Discharge Machining}

The addition of semi conductive particles in a dielectric fluid is termed as Powder Mixed Electric Discharge Machining. The mixing of fine powders of Aluminum, Silicon, and Graphite with dielectric solution enhances the machined surface properties. Regardless of its strength, hardness, toughness, and microstructure, any material that is electrically conductive can be machined.[2]

\section{RESEARCH PROGRESS IN POWDER MIXED ELECTRIC DISCHARGE MACHINING}

For enhancing the machined surface properties in EDM, fine powders of Aluminum, Silicon and Graphite are blended with a dielectric solution. In this section, the work was done by different researchers in recent years from 2015 to 2018 on the usage of different powders and their effects in EDM is discussed.

Rajesh Bajaj (2015), reviewed different research papers and presented a summary of powder mixed EDM by mixing nano and micro-sized particles in a dielectric fluid with various combinations of tool electrode and workpiece. Enhanced surface finish was achieved with decrease in particle size and increase in powder concentration. The electrode gap is increased twice due to less heat transfer to work piece by micro or nanoparticles as compared to non PMEDM, thereby improving surface finish and reducing tool wear rate.[3]

Gangadharudu Talla (2015), fabricated and machined Aluminum MMC by adding the Aluminum powder in the dielectric. The results depicted that MRR is better and there was a notable decrease in Surface Roughness Ra. A hybrid approach of dimensional and regression analysis had been established. The results depicted that coefficient of expansion, thermal conductivity, a density of material also affect significantly both MRR and Surface Roughness.[4]

Chander Prakash (2015), suggested a unique biometric non-porous bioceramic surface on Beta Titanium ( $\beta$-Ti) alloy for orthopedic applications by Powder Mixed EDM. Different tests were conducted before machining, after EDM, and after Powder Mixed EDM. By observing scanning electron microscope (SEM) images, the non-machined surface displayed a relatively smooth surface, Energy-dispersive X-ray spectroscopy (EDS) point analysis confirmed the presence of Titanium (Ti), Columbium (Nb), Tantalum (Ta), Zirconium ( $\mathrm{Zr}$ ) and Oxygen (O). The electric discharge machined surface featured cracks, wide and deep craters. In order to reduce these craters and micro-cracks, used Silicon powder particles mixed in oil as the dielectric solvent, which also minimized the obtaining of crater characteristics. The machining gap was increased that led to a reduction in the production of thermal energy by electrical sparks due to the Silicon powder presence in the dielectric fluid. The microhardness of Powder Mixed EDMed surface was more by $184.42 \%$ than that of non treated surface of Beta Titanium $\beta$-Ti alloy. The improvement in hardness of machined surface hardness is due to the long interval of pulse time (pulse off time). Bone anchoring and biochemical stability both are favored by a rough implant, whereas resistance to abrasion wear is provided by a hard surface.[5]

Murahari Kolli (2015), employed a Taguchi method for optimizing the concentration of surfactant and graphite powder mixed in dielectric fluid for EDM of alpha-beta titanium alloy Ti-6Al-4V. Material Removal Rate was improved significantly, reduced Tool Wear Rate, Recast Layer Thickness and Surface Roughness at various conditions. A concentration of graphite powder effected RLT, SR, and Discharge Current Surfactant effected T and Material Removal Rate.[6]

V. Vikram Reddy (2015), utilized Grey Relational Analysis to optimize process parameters of EDM process using surfactant and graphite powder mixed dielectric. The workpiece material was PH 17-4 Stainless Steel and tool was Copper. 
From the results, it was determined that machining performance had been improved considerably. Various confirmation experiments were performed by using optimum parametric setting. It was found that the responses at parameters of the optimal combination are in good agreement with the results of confirmation experiments.[7]

Hamid Baseri (2015), investigations were made to determine the effect of Titanium Dioxide $\left(\mathrm{TiO}_{2}\right)$ nano-powder, its powder concentration, spark energy and tool rotational speed on Surface Roughness, tool wear rate, and material removal rate. From the results, it was discerned that MRR, SR, TWR were increasing. Tool wear rate was increased when tool rotational speed was increased. Surface roughness was decreased since the debris exit better from the machining gap.[8]

Houriyeh Marashi (2015), investigations were done to increase characteristics of AISI D2 Steel machined using Titanium (Ti) nanopowder added to dielectric varying discharge duration and peak current. The surface morphology, surface finish, and MRR were enhanced by adding Titanium nanopowder. There was negligible deposition of Titanium and the atomic concentration of Titanium was increased around crack areas.[9]

Balbir Singh (2015), investigated tool wear rate in PMEDM of aluminum 6061 alloy reinforced with $10 \%$ Silicon Carbide particles by adding Tungsten powder in a dielectric fluid. The process parameters Pulse on time, Current, Pulse off time and gap voltage were optimized utilizing Response Surface Methodology approach. The TWR was reduced by about $51.12 \%$ by using PMEDM when compared with conventional EDM process. Tool wear rate increases significantly with a rise in the current whereas a higher pulse on time yields a moderate reduction in tool wear rate.[10]

Sanjeev Kumar (2015), titanium alloys were machined in the EDM process utilizing Tungsten powder and Manganese powder separately. The results of this experimental study were utilized for developing a micro mathematical model for predicting TWR by considering thermophysical properties of tool materials and significant parameters of EDM. The factor which is most significantly affecting tool wear rate was Peak Current followed by pulse-on-time and tool material. Adding Manganese powder in dielectric showed the higher effect when compared with Tungsten powder.[11]

Bouchmel Mliki (2015), used Lattice Boltzmann Method to numerically investigate heat transfer and fluid flow inside the enclosure of L-shape filled with Copper/water nanofluid. The obtained results depicted that the amount of heat transfer is enhanced by nanofluid and aspect ratio reduction enhances this effect. Also, it was established that with a rise in Rayleigh number there is a rise in the mean Nusselt number.[12]

Nihal Ekmekci (2015), used a mixture of Hydroxyapatite (HA) powder and deionized water as a dielectric liquid during EDM of alpha-beta titanium alloy Ti6Al4V work material. The results indicated the possibility of Hydroxyapatite deposition on titanium alloy surfaces by the use of the process. It is possible to switch between the deposition and removal process during the machining and suggest an attractive method for arrangement the shape of the substrate and thickness of the covering Hydroxyapatite layer for future applications.[13]

Houriyeh Marashia (2016), had discussed the employment of different powders in Powder Mixed EDM. The machining efficiency is influenced by powder particles such as shape, size, and concentration in the dielectric. Graphite \& Aluminum powders improved the efficiency concerning surface quality and MRR. Molybdenum disulfide $\left(\mathrm{MoS}_{2}\right) \&$ Graphite powders improved lubricity. Copper \& crushed Glass did not influence machining efficiency while Copper powders were effective in surface modification. Powder concentrations below $20 \mathrm{~g} / \mathrm{l}$ had been used. 1 to $55 \mu \mathrm{m}$ micro-sized powders and 20 to $150 \mathrm{~nm}$ nano-sized powders were used.[14] 
Gangadharudu Talla (2016), furnished a detailed review of the ongoing research in Powder Mixed EDM. High thermal and electrical conductivities, low density are the ideal combination for producing good machining rate \& higher surface quality. Due to ease of availability, less price and good results, Silicon, Aluminum and Silicon Carbide are widely used. Carbon nanotubes produce spectacular results but rarely used due to high cost. In finish machining, better results are produced by positive polarity, powder material deposition on the workpiece is also more. For rough machining operations, negative polarity is preferred.[15]

Gangadharudu Talla (2016), investigated the consequence of usage of different powders aluminum, silicon, graphite on different surface integrity characteristics while machining Inconel 625. To reduce the number of surface cracks Graphite was suggested, for fine surface finish, high microhardness Silicon was suggested. Aluminum follows the Silicon for finer surface finish. But at higher concentrations of powder (above 4g/L), Graphite was suggested compared to Aluminum particles.[16]

Chander Prakash (2016), Beta Titanium $\beta$-Ti implant had been machined used powder mixed EDM. It was concluded that mixing of Silicon powder in dielectric fluid improved the surface quality by reduction of defects like craters, recast layer thickness, microcracks. And also enhanced TWR and MRR at same working conditions.[17]

Chander Prakash (2016), fatigue performance of powder mixed EDM specimen is improved, cell adhesion and proliferation were promoted. Powder mixed EDM enhanced the mechanical properties and osteoblast response.[18]

Ahmed Al-Khazraji (2016), concluded that the graphite electrode with Silicon Carbide powder dielectric improved the fatigue safety factor by $7.3 \%$ in comparison with a copper electrode with the same dielectric and by $18.61 \%$ $\& 14.61 \%$, in comparison with results employing Kerosene dielectric with Graphite and Copper electrodes respectively.[19]

Beulent Ekmekci (2016), machined Titanium alloy Ti6Al4V work material utilizing a mixture of Silicon Carbide powder and de-ionized water by varying pulse currents and pulse on duration. The results showed the minimum deformation of the machined surface in regard to roughness, microcracks, and thickness of heat affected layer. The material transfer rate was increased due to the generation of secondary discharges which are unevenly separated.[20]

Jagdeep Singh (2016), graphite powder was used in EDM of Tungsten Carbide alloy. The multi-performance characteristics i.e. micro-hardness \& surface roughness were optimized. The Tungsten Carbide alloy's surface characteristics were improved by the use of graphite powder. The dominating factor was pulse-on time compared to other factors.[21]

Gangadharudu Talla (2016), investigated various surface defects during EDM of Inconel 625 utilizing dielectric with graphite powder suspension. Due to the formation of large and shallow craters surface finish was improved. Due to the reduction in discharge energy density, white layer thickness was decreased. In the subsurface region, microhardness was declined. Surface tensile stress was decreased which in turn improved the fatigue strength.[22]

Ryota Toshimitsua (2016), surface finish characteristics by using a mixture of chromium powder and fluid in the EDM process were discussed experimentally. When there was sufficient stirring of chromium powder mixed fluid (CrPMF), there is an achievement of small surface roughness by electric discharge finishing. When there is low discharge current and high chromium powder concentration, there was an increase in the content of chromium on the surface. When chromium powder concentration and discharge current was higher, the layer that contains chromium becomes thick. 
There was a rise in the Vickers hardness, corrosion resistance and water repellency of electric discharge machining finished surface.[23]

S. Tripathy (2016), chromium powder was added to dielectric fluid EDM of H-11 die steel. Determination of significant process variables set was done by multi-attribution optimization utilizing TOPSIS and GRA. ANOVA was done. The surface roughness was reduced by adding proper concentration and size of powder particles. There is an improvement in surface topography with minimal defects, surface roughness, and cracks by mixing of conductive powder to dielectric fluid. Powder concentration (Cp), Duty Cycle (DC), Pulse on Time (Ton) and Peak Current (Ip) are significant parameters contributing towards the improvement of preference solution while Gap Voltage (Vg) is insignificant.[24]

Sagar Patel (2017), Inconel was machined using a mixture of Aluminum oxide $\left(\mathrm{Al}_{2} \mathrm{O}_{3}\right)$ powder with a particle concentration of $0.5-1.5 \mathrm{gm} / 1$ into the dielectric. The conclusions deduced were peak current and duty cycle are critical for material removal rate, peak current, slurry concentration, duty cycle, and voltage are critical for tool wear rate, peak current, ON time and duty cycle for Surface Roughness. The concentration of powders retards the deposition of Aluminum oxide $\left(\mathrm{Al}_{2} \mathrm{O}_{3}\right)$ particles on workpiece.[25]

Shalini Mohanty (2017), Aluminum oxide $\left(\mathrm{Al}_{2} \mathrm{O}_{3}\right)$ powders suspended in commercial grade EDM oil is used as the dielectric fluid. The workpiece material is $\mathrm{Al}-\mathrm{SiCp} \mathrm{12 \%} \mathrm{metal} \mathrm{matrix} \mathrm{composite.} \mathrm{The} \mathrm{results} \mathrm{discerned} \mathrm{that} \mathrm{the} \mathrm{powders}$ enhanced the machining rate and surface finish. Statistical validation of mathematical models developed utilizing Response Surface Methodology also indicated the significant variation in surface finish and material removal rate.[26]

Amit Kumar (2017), the EDM process is done on Inconel 825 alloy by Aluminum oxide $\left(\mathrm{Al}_{2} \mathrm{O}_{3}\right)$ nanopowder suspension in deionized water. The results revealed that in comparison with convention EDM, MRR \& SR is higher by about $44 \%$ and $55 \%$ respectively. Due to the rise in spark gap between electrodes, resulted in better flushing, lesser short-circuiting and longer machining time, as a consequence, higher machining stability is obtained for nanopowder mixed EDM. Surface topography of machined surface is improved significantly using Nanopowder mixed EDM (NPMEDM). Microcracks were reduced.[27]

Vijay Kumar (2017), developed a finite element model to predict the Beryllium Copper alloy material removal rate machined by PMEDM process utilizing suspension of powder of Aluminum oxide in the dielectric with a particle mesh size of $150 \mu$. Gap current, powder concentration was varied, 12 experiments were conducted practically and compared with numerical methods. The results revealed that a $7.8 \%$ average percentage error was obtained between numerical and experimental values. So, before practically machining a workpiece the finite element model can be considered to predict the material removal rate, which in turn saves experimentation cost and time.[28]

S. Tripathy (2017), machined H-11 die steel by suspending Silicon Carbide powder in dielectric fluid taking Copper as the electrode. As powder concentration is increased surface texture showed significant enhancement due to an increase in material removal rate, reduction in RLT, surface roughness, enhanced microhardness with less microcrack.[29]

Tien-Long Banh (2017), GRA and Taguchi method were performed to optimize workpiece material, polarity, tool material, current, Titanium powder concentration, pulse-on time and pulse-off time. The optimal results from both methods showed that there is reduction of SR and TWR, while there is a rise in MRR and microhardness of surface.[30]

Pallavi Chaudhury (2017), EN 19 steel was electrical discharge machined with adding tungsten powder mixed in the dielectric. Peak current, tungsten powder concentration, and pulse on time were the influential parameters of PMEDM. 
Larger MRR was achieved, TWR was increased initially and then decreased with a rise in Current \& Pulse on time.[31]

Mohammadreza Shabgard (2017), used fluid of carbon nanotube adding into dielectric for machining Ti-6Al-4V alloy in EDM process. Considerable improvement in machining stability is achieved by adding multiwall carbon nanotubes into dielectric due to the reduction in inappropriate sparks mainly during long pulse on times and low energy pulses. MRR was reduced, but MRR is increased in long pulse durations in association with low currents. In PMEDM process in shorter pulse on times, Tool wear rate reduces and this reduction is very important. In stable machining, by usage of Carbon nanotube added dielectric the EDMed work-pieces SR is lower than the workpieces surface roughness machined by using conventional dielectric. Contrary in unstable conditions sparking stability is increased by use of carbon nanotubes, consequently, surface roughness enhances by the increase MRR. Addition of carbon nanotube particles into dielectric decreases the length and the size of surface microcracks.[32]

Soumen Mandal (2017), focused on the suitability of synthesizing silver (Ag) and molybdenum disulfide $\left(\mathrm{MOS}_{2}\right)$ nanoparticle in dielectric dimethylformamide solution for micro-electro-discharge machining. The results and inferences indicated that use of synthesized nanoparticle dielectric in micro- EDM enhances the MRR by 2 to 3 times and spark gap by two times while maintaining uniformity in distribution of sparks gap energy. Moreover, an increase in spark gap enhances the stability of micro-electric discharge machining process by eliminating unnecessary arcing phenomenon. Additionally, higher and uniform spark gap energy steers to larger MRR and better shape of the machined features.[33]

Tahsin T. Öpöz (2018), investigated the Silicon Carbide powder concentration impact on particles deposition, surface topography and subsurface structures in PMEDM of Titanium alloy Ti-6Al-4V- Extra Low Interstitial (ELI) work material. The material transfer mechanism was enhanced at high concentration of the suspended particle in dielectric liquid and low pulse currents. With an increase of Silicon Carbide powder, concentration decreased the re-solidified layer thicknesses. But the layer hardness was increased.[34]

Santosh Kumar Sahu (2018), Inconel 718 was machined using the EDM process by using a mixture of graphite powder and dielectric media by varying peak discharge current. The results showed there was an improvement in MRR, superior surface finish, reduction in TWR and reduction in intensity \& severity of surface cracking. Lesser residual stress and microhardness were achieved, reduced the hard carbide layers formation.[35]

N A J Hosni (2018), AISI D2 hardened steels surface characteristics were investigated by powder mixed EDM utilizing micro/nano Chromium powder in the dielectric. The recast layer in powder mixed EDM was enhanced by as high as $41-53 \%$ in comparison with conventional EDM. There is a reduction in the RLT significantly in nano-Chromium size with a combination of the span - 20 surfactant.[36]

\section{CONCLUDING REMARKS}

A review has been presented on the work done by different authors in powder mixed electric discharge machining, the effect of different powders on material removal rate, surface finish, tool wear rate, recast layer thickness, craters, microcracks and micro-hardness.

The following conclusions can be made from this review:

- PMEDM offers better results in enhancing material removal rate, surface finish and reduction in tool wear rate and recast layer thickness. 
- PMEDM also reduces craters, microcracks and increases microhardness.

- Due to ease of availability, less price and good results, Silicon, Aluminum and Silicon Carbide are widely used. Carbon nanotubes produce spectacular results but rarely used due to high cost.

\section{FUTURE SCOPE}

- Little work has been done on the use of nanopowders in powder mixed electric discharge machining. So there is need of in-depth investigation of the applicability of nanopowders in PMEDM.

- Machining of Nickel alloys using PMEDM also needs further attention.

\section{REFERENCES}

1. "How Sinker EDM Machining Works.".

2. S. Boral, "Powder Mixed Electric Discharge Machining.".

3. R. Bajaj, A. K. Tiwari, and A. R. Dixit, "Current Trends in Electric Discharge Machining Using Micro and Nano Powder Materials- A Review,” Mater. Today Proc., vol. 2, no. 4-5, pp. 3302-3307, 2015.

4. G. Talla, D. K. Sahoo, S. Gangopadhyay, and C. K. Biswas, "Modeling and multi-objective optimization of powder mixed electric discharge machining process of aluminum/alumina metal matrix composite," Eng. Sci. Technol. an Int. J., vol. 18, no. 3, pp. 369-373, 2015.

5. C. Prakash, H. K. Kansal, B. S. Pabla, and S. Puri, "Processing and Characterization of Novel Biomimetic Nanoporous Bioceramic Surface on $\beta$-Ti Implant by Powder Mixed Electric Discharge Machining," J. Mater. Eng. Perform., vol. 24, no. 9, pp. 3622-3633, 2015.

6. M. Kolli and A. Kumar, "Engineering Science and Technology, an International Journal Effect of dielectric fl uid with surfactant and graphite powder on Electrical Discharge Machining of titanium alloy using Taguchi method," Eng. Sci. Technol. an Int. J., vol. 18, no. 4, pp. 524-535, 2015.

7. V. V. Reddy, C. S. Reddy, and P. M. Valli, "Optimization of Process Parameters of Surfactant and Graphite Powder Mixed Dielectric EDM through Taguchi-Grey Relational Analysis," vol. 5, no. 4, pp. 175-180, 2015.

8. H. Baseri and S. Sadeghian, "Effects of nanopowder TiO 2 -mixed dielectric and rotary tool on EDM," 2015.

9. H. Marashi, A. A. D. Sarhan, and M. Hamdi, "Applied Surface Science Employing Ti nano-powder dielectric to enhance surface characteristics in electrical discharge machining of AISI D2 steel,” Appl. Surf. Sci., vol. 357, pp. 892-907, 2015.

10. P. Taylor, B. Singh, J. Kumar, and S. Kumar, "Materials and Manufacturing Processes Investigation of the Tool Wear Rate in Tungsten Powder Mixed Electric Discharge Machining of AA6061 / 10 \% SiC p Composite," no. April 2015, pp. 37-41.

11. S. Kumar, R. Singh, A. Batish, and T. P. Singh, "Modeling the tool wear rate in powder mixed electro-discharge machining of titanium alloys using dimensional analysis of cryogenically treated electrodes and workpiece," vol. 0, no. 0, pp. 1-12, 2015.

12. B. Mliki, M. Ammar, K. Guedri, and A. Omri, "Engineering Science and Technology, an International Journal Lattice Boltzmann simulation of natural convection in an L-shaped enclosure in the presence of nano fl uid," Eng. Sci. Technol. an Int. J., vol. 18, no. 3, pp. 503-511, 2015.

13. N. Ekmekci and B. Ekmekci, "Electrical Discharge Machining of Ti6Al4V in Hydroxyapatite Powder Mixed Dielectric Liquid," vol. 6914, no. January, pp. 0-26, 2016. 
14. H. Marashi, D. M. Jafarlou, A. A. D. Sarhan, and M. Hamdi, "State of the art in powder mixed dielectric for EDM applications," Precis. Eng., vol. 46, pp. 11-33, 2016.

15. G. Talla, S. Gangopadhayay, and C. K. Biswas, "State of the art in powder-mixed electric discharge machining: A review," Proc. Inst. Mech. Eng. Part B J. Eng. Manuf., vol. 231, no. 14, pp. 2511-2526, 2017.

16. G. Talla, S. Gangopadhyay, and C. K. Biswas, "Effect of impregnated powder materials on surface integrity aspects of Inconel 625 during electrical discharge machining,” Proc. Inst. Mech. Eng. Part B J. Eng. Manuf., vol. 232, no. 7, pp. 1259-1272, 2018.

17. C. Prakash, H. K. Kansal, B. S. Pabla, and S. Puri, "Experimental investigations in powder mixed electric discharge machining of Ti-35Nb-7Ta-5Zrß-titanium alloy,” Mater. Manuf. Process., vol. 32, no. 3, pp. 274-285, 2017.

18. Nagaral, M., Auradi, V., \& Ravishankar, M. K. (2013). mechanical behaviour of aluminium 6061 alloy reinforced with al2o3 \& graphite particulate hybrid metal matrix composites. International Journal of Research in Engineering \& Technology (IJRET) Vol, 1, 193-198.

19. C. Prakash, H. K. Kansal, B. S. Pabla, and S. Puri, "Powder Mixed Electric Discharge Machining: An Innovative Surface Modification Technique to Enhance Fatigue Performance and Bioactivity of $\beta$-Ti Implant for Orthopedics Application,” J. Comput. Inf. Sci. Eng., vol. 16, no. 4, p. 041006, 2016.

20. A. Al-Khazraji, S. A. Amin, and S. M. Ali, "The effect of SiC powder mixing electrical discharge machining on white layer thickness, heat flux and fatigue life of AISI D2 die steel," Eng. Sci. Technol. an Int. J., vol. 19, no. 3, pp. 1400-1415, 2016.

21. H. Yas and N. Ekmekci, “A Discharge Separation Model for Powder Mixed Electrical Discharge Machining,” vol. 138, no. August 2016, pp. 1-9, 2017.

22. J. Singh and R. K. Sharma, “Ac ce p te d us t," Perspect. Sci., 2016.

23. G. Talla, S. Gangopadhyay, and C. K. Biswas, "Influence of Graphite Powder Mixed EDM on the Surface Integrity Characteristics of Inconel 625 Influence of Graphite Powder Mixed EDM on the Surface Integrity Characteristics of Inconel 625," vol. 6351, no. March, 2016.

24. R. Toshimitsu, A. Okada, R. Kitada, and Y. Okamoto, "Improvement in Surface Characteristics by EDM with Chromium Powder Mixed Fluid,” Procedia CIRP, vol. 42, no. Isem Xviii, pp. 231-235, 2016.

25. S. Tripathy and D. K. Tripathy, "Engineering Science and Technology, an International Journal Multi-attribute optimization of machining process parameters in powder mixed electro-discharge machining using TOPSIS and grey relational analysis," Eng. Sci. Technol. an Int. J., vol. 19, no. 1, pp. 62-70, 2016.

26. S. Patel, D. Thesiya, and A. Rajurkar, "Aluminium powder mixed rotary electric discharge machining (PMEDM) on Inconel 718,” Aust. J. Mech. Eng., vol. 16, no. 1, pp. 21-30, 2018.

27. S. Mohanty, A. Mishra, B. K. Nanda, and B. C. Routara, "Multi-objective parametric optimization of nano powder mixed electrical discharge machining of AlSiCpusing response surface methodology and particle swarm optimization," Alexandria Eng. J., pp. 2-12, 2016.

28. A. Kumar, A. Mandal, A. R. Dixit, and A. K. Das, "Performance evaluation of Al2O3nano powder mixed dielectric for electric discharge machining of Inconel 825,” Mater. Manuf. Process., vol. 33, no. 9, pp. 986-995, 2018.

29. V. S. Jatti and S. Bagane, "Thermo-electric modelling, simulation and experimental validation of powder mixed electric discharge machining (PMEDM) of BeCu alloys,” Alexandria Eng. J., 2017.

30. S. Tripathy and D. K. Tripathy, "Surface Characterization and Multi-response optimization of EDM process parameters using 
powder mixed dielectric," Mater. Today Proc., vol. 4, no. 2, pp. 2058-2067, 2017.

31. T. Banh, H. Nguyen, and C. Ngo, "Characteristics optimization of powder mixed electric discharge machining using titanium powder for die steel materials," vol. 0, no. 0, pp. 1-18, 2017.

32. P. Chaudhury, S. Samantaray, and S. Sahu, "ScienceDirect Multi Response Optimization of Powder Additive Mixed Electrical Discharge Machining by Taguchi Analysis,” Mater. Today Proc., vol. 4, no. 2, pp. 2231-2241, 2017.

33. M. Shabgard and B. Khosrozadeh, "Investigation of carbon nanotube added dielectric on the surface characteristics and machining performance of Ti-6Al-4V alloy in EDM process,” J. Manuf. Process., vol. 25, pp. 212-219, 2017.

34. Dinesh, S., Antony, A. G., Rajaguru, K., \& Parameswaran, P. (2018). Comprehensive analysis of wire electric discharge machining process in machining high chromium high carbon steel. International Journal of Mechanical and Production Engineering Research and Development (IJMPERD), 8(1), 65-74.

35. S. Mandal and R. V. Kumar, "Silver and molybdenum disulfide nanoparticles synthesized in situ in dimethylformamide as dielectric for micro-electro discharge machining," 2017.

36. T. T. Öpöz, H. Yas, N. Ekmekci, and B. Ekmekci, "Particle migration and surface modification on Ti6Al4V in SiC powder mixed electrical discharge machining," vol. 31, pp. 744-758, 2018.

37. S. K. Sahu and S. Datta, "Experimental studies on graphite machining of Inconel 718 super alloys: Comparison with conventional electro-discharge machining," vol. 0, no. 0, pp. 1-19, 2018.

38. A. A. Khan, M. Binti, and A. Bakar, "The influence of Span-20 surfactant and micro- / nano-Chromium ( Cr ) Powder Mixed Electrical Discharge Machining ( PMEDM) on the surface characteristics of AISI D2 hardened steel The influence of Span20 surfactant and micro- / nano- Chromium ( Cr ) Powder Mixed Electrical Discharge Machining ( PMEDM) on the surface characteristics of AISI D2 hardened steel." 
